\title{
COMPLETELY REDUCIBLE LIE SUBALGEBRAS
}

\author{
GEORGE MCNINCH
}

\begin{abstract}
Let $G$ be a connected and reductive group over the algebraically closed field $K$. J-P. Serre has introduced the notion of a $G$-completely reducible subgroup $H \subset G$. In this note, we give a notion of $G$-complete reducibility - $G$-cr for short - for Lie subalgebras of $\operatorname{Lie}(G)$, and we show that if the closed subgroup $H \subset G$ is $G$-cr, then $\operatorname{Lie}(H)$ is $G$-cr as well.
\end{abstract}

\section{INTRODUCTION}

Let $G$ be a connected and reductive group over the algebraically field $K$, and write $\mathfrak{g}$ for the Lie algebra of $G$. J-P. Serre has introduced the notion of a $G$-completely reducible subgroup; we state the definition here only for a closed subgroup $H \subset G$. We say $H$ is $G$-cr provided that whenever $H \subset P$ for a parabolic subgroup of $G$, there is a Levi factor $L \subset P$ such that $H \subset L$; cf. Ser 05. When $G=\mathrm{GL}(V)$, the subgroup $H$ is $G$-cr if and only if $V$ is a semisimple $H$-module. Similarly, if the characteristic of $K$ is not 2 and $G$ is either the symplectic group $\operatorname{Sp}(V)$ or the orthogonal group $\mathrm{SO}(V)$, a subgroup $H$ of $G$ is $G$-cr if and only $V$ is a semisimple $H$-module.

B. Martin Ma 03 used some techniques from "geometric invariant theory" - due to G. Kempf and to G. Rousseau - to prove that if $H \subset G$ is $G$-cr, and if $N$ is a normal subgroup of $H$, then $N$ is $G$-cr as well; cf. Ser 05, Théorème 3.6]. Martin's result was obtained first for strongly reductive subgroups in the sense of Richardson; it follows from BMR 05 that the strongly reductive subgroups of $G$ are precisely the $G$-cr subgroups. See also Ser 05] §3.3] for an overview of these matters.

We are going to prove in this note a result related to that of Martin. If $\mathfrak{h} \subset \mathfrak{g}$ is a Lie subalgebra, say that $\mathfrak{h}$ is $G$-cr provided that whenever $\mathfrak{h} \subset \operatorname{Lie}(P)$ for a parabolic subgroup $P$ of $G$, there is a Levi factor $L \subset P$ such that $\mathfrak{h} \subset \operatorname{Lie}(L)$.

We will prove:

Theorem 1. Suppose that $G$ is a reductive group over the algebraically closed field $K$.

(1) Let $X_{1}, \ldots, X_{d}$ be a basis for the Lie subalgebra $\mathfrak{h} \subset \mathfrak{g}$. Then $\mathfrak{h}$ is $G$-cr if and only if the $\operatorname{Ad}(G)$-orbit of $\left(X_{1}, \ldots, X_{d}\right)$ is closed in $\bigoplus^{d} \mathfrak{g}$.

(2) If the closed subgroup $H \subset G$ is $G$-cr, then $\operatorname{Lie}(H)$ is $G$-cr as well.

Our result - and our techniques - are related to those used by Richardson in $\mathrm{Ri} 88$, though he treats mainly the case of characteristic 0 . See e.g. loc. cit. Theorem 3.6.

The converse to Theorem 1(2) is not true. Indeed, suppose the characteristic $p$ of $K$ is positive, and consider a finite subgroup $H \subset G$ whose order is a power of $p$. Then $\operatorname{Lie}(H)=0$ is clearly $G$-cr; however, if $G=\mathrm{SL}(V)$ and if $H$ is non-trivial, then $V$ is not semisimple as an $H$-module, thus $H$ is not $G$-cr. The converse to Theorem 12) is even false for connected $H$; I thank Ben Martin for pointing out the following example. Take for $H$ any semisimple group

Date: December 30, 2005.

Research of the author supported in part by the US National Science Foundation through DMS-0437482. 
in characteristic $p>0$, let $\rho_{i}: H \rightarrow \mathrm{SL}\left(V_{i}\right)$ be representations for $i=1,2$ with $\rho_{1}$ semisimple and $\rho_{2}$ not semisimple, and consider the representation $\rho: H \rightarrow G=\operatorname{SL}\left(V_{1} \oplus V_{2}\right)$ given by $h \mapsto \rho_{1}(h) \oplus \rho_{2}(F(h))$ where $F: H \rightarrow H$ is the Frobenius endomorphism. If $J$ denotes the image of $\rho$, then $J$ is not $G$-cr since $V_{1} \oplus V_{2}$ is not semisimple as a $J$-module. However, $\operatorname{Lie}(J)=\operatorname{im} d \rho$ lies in the the Lie algebra of the subgroup $M=\operatorname{SL}\left(V_{1}\right) \times \operatorname{SL}\left(V_{2}\right)$; moreover, $M$ is a Levi factor of a parabolic subgroup of $G$, and $\operatorname{Lie}(J)=\operatorname{im} d \rho_{1} \oplus 0 \subset \mathfrak{s l}\left(V_{1}\right) \oplus \mathfrak{s l}\left(V_{2}\right)=$ Lie $(M)$. Since the image of $\rho_{1} \times 1: H \rightarrow M$ is $M$-cr (use BMR 05 Lemma 2.12(i)]), the main result of this paper implies Lie $(J)$ to be $M-\mathrm{cr}^{1}$, hence Lemma 4 below shows that Lie $(J)$ is $G$-cr as well.

The author would like to thank Michael Bate, Benjamin Martin, and Gerhard Röhrle for some comments and conversations which were useful in preparing this note. He would also like to acknowledge the hospitality of the Centre Interfacultaire Bernoulli at the École Polytechnique Fédérale de Lausanne during a visit in June 2005 during which a need for the result of this note became clear to the authors of [MT 05].

\section{Preliminaries}

We work throughout in the geometric setting; thus, $K$ is an algebraically closed field. A variety will mean a separated and reduced scheme of finite type over $K$. The group $G$ will be a connected and reductive algebraic group (over $K$ ). A closed subgroup $H \subset G$ is in particular a subvariety of $G$ and so $H$ is necessarily reduced - e.g. if $G$ acts on a variety $X$ and if $x \in X$, then $\operatorname{Stab}_{G}(x)$ will mean the reduced subgroup determined by the "abstract group theoretic" stabilizer (even if the $G$-orbit of $x$ is not separable).

2.1. Closed orbits. Let $X$ be an affine $G$-variety, let $x \in X$ and choose a maximal torus $S \subset \operatorname{Stab}_{G}(x)$ of the stabilizer in $G$ of $x$. Let $L=C_{G}(S)$; thus $L$ is a Levi factor of a parabolic subgroup of $G$.

Proposition 2. If the $G$-orbit $G \cdot x$ is closed in $X$, then the $L$-orbit $L \cdot x$ is closed in $X$.

Proof. The fixed point set $X^{S}$ is closed in $X$. Since by assumption $G \cdot x$ is closed in $X$, it follows that

is closed in $X$.

$$
(G \cdot x)^{S}=X^{S} \cap G \cdot x
$$

Let now $N=N_{G}(S)$ be the normalizer in $G$ of $S$. We claim that $(G \cdot x)^{S}=N \cdot x$. Indeed, let $g \in G$ and suppose $g \cdot x$ is fixed by $S$. The claim follows once we prove that $g \cdot x \in N \cdot x$. Well, for each $s \in S$ we have $s g \cdot x=g \cdot x$ so that $g^{-1} s g \in \operatorname{Stab}_{G}(x)$. Thus $g^{-1} S g$ is a maximal torus of $\operatorname{Stab}_{G}(x)$. Since maximal tori are conjugate [Spr 98, Theorem 6.4.1], there is an element $h \in \operatorname{Stab}_{G}(x)$ such that $g^{-1} S g=h S h^{-1}$. But then $g h \in N$, and moreover, $g \cdot x=g h \cdot x$.

Note that $N$ contains $L$ as a normal subgroup. We now observe that the stabilizer in $L$ of a point $y$ of the orbit $N \cdot x$ is conjugate to $\operatorname{Stab}_{L}(x)$ by an element of $N$. Indeed, choosing $h \in N$ such that $h \cdot x=y$, one knows that $h \cdot \operatorname{Stab}_{L}(y) \cdot h^{-1}=\operatorname{Stab}_{L}(x)$. It follows that all $L$-orbits in $N \cdot x$ have the same dimension.

Since the closure of any $L$-orbit must be the union of orbits of strictly smaller dimension, it follows that the $L$-orbits in $N \cdot x$ are closed.

Since $N \cdot x=X^{S} \cap G \cdot x$ is closed in $X$, it follows at once that $L \cdot x$ is closed in $X$, as required.

\footnotetext{
${ }^{1}$ This can be seen more easily: it is straightforward to check that a Lie subalgebra $\mathfrak{h} \subset \mathfrak{s l}(V)$ is $\operatorname{SL}(V)$-cr if and only if $V$ is a semisimple $\mathfrak{h}$-module.
} 
Remark 3. With notation as in the previous Proposition, if $N=N_{G}(S)$, it follows from the rigidity of tori Spr 98, Corollary 3.2.9] that $L$ has finite index in $N$. In particular, $(G \cdot x)^{S}$ is a finite union of $L$-orbits which are permuted transitively by $N$; moreover, these $L$-orbits are precisely the connected components of $(G \cdot x)^{S}$.

2.2. Complete reducibility. The interpretation of complete reducibility using the spherical building of $G$ permits one to prove the following:

Lemma 4. Let $G$ be reductive and let $M \subset G$ be a Levi factor of a parabolic subgroup of $G$. Suppose that $J \subset M$ is a subgroup, and that $\mathfrak{h} \subset \operatorname{Lie}(M)$ is a Lie subalgebra. Then $J$ is $G$-cr if and only if $J$ is $M$-cr and $\mathfrak{h}$ is $G$-cr if and only if $\mathfrak{h}$ is $M$-cr.

Proof. The assertion for $J$ follows from [Ser 05, Proposition 3.2]. The proof for $\mathfrak{h}$ is similar; let us give a sketch. Write $X$ for the building of $G$. The Lie subalgebra $\mathfrak{h}$ defines a subcomplex $Y$ of $X$ : the simplices of $Y$ are those simplices in $X$ which correspond to parabolic subgroups $P$ with $\mathfrak{h} \subset \operatorname{Lie}(P)$.

Recall [Bo 91, Corollary 14.13] that the intersection $P \cap P^{\prime}$ of two parabolic subgroups $P, P^{\prime} \subset G$ contains a maximal torus of $G$. This implies that $\operatorname{Lie}\left(P \cap P^{\prime}\right)=\operatorname{Lie}(P) \cap \operatorname{Lie}\left(P^{\prime}\right)$; see e.g. the argument in the first paragraph of [Ja 04, $\S 10.3]$.

If now $\mathfrak{h} \subset \operatorname{Lie}(P) \cap \operatorname{Lie}\left(P^{\prime}\right)$, it follows that $\mathfrak{h} \subset \operatorname{Lie}\left(P \cap P^{\prime}\right)$. This shows that the subcomplex $Y$ is convex; see Ser 05 Prop. 3.1]. Evidently $\mathfrak{h}$ is $G$-cr if and only if $Y$ is $X$-cr in the sense of [Ser 05, §2.2].

Choose a parabolic subgroup $Q$ for which $M$ is a Levi factor. Then we may identify the building of $M$ with the residual building of $X$ determined by the parabolic $Q$; cf. [Ser 05] 2.1.8 and 3.1.7]. Now the claim follows from [Ser 05 Proposition 2.5].

2.3. Cocharacters and parabolic subgroups. If $V$ is a variety and $f: \mathbf{G}_{m} \rightarrow V$ is a morphism, we write $v=\lim _{t \rightarrow 0} f(t)$, and we say that the limit exists, if $f$ extends to a morphism $\tilde{f}: \mathbf{A}^{1} \rightarrow V$ with $\tilde{f}(0)=v$. If $\mathbf{G}_{m}$ acts on $V$, a closed point $w \in V$ determines a morphism $f: \mathbf{G}_{m} \rightarrow V$ via the rule $t \mapsto t \cdot w$; one writes $\lim _{t \rightarrow 0} t \cdot w$ as shorthand for $\lim _{t \rightarrow 0} f(t)$.

A cocharacter of an algebraic group $A$ is a $K$-homomorphism $\gamma: \mathbf{G}_{m} \rightarrow A$. A linear $K$-representation $(\rho, V)$ of $A$ yields a linear $K$-representation $(\rho \circ \gamma, V)$ of $\mathbf{G}_{m}$. Then $V$ is the direct sum of the weight spaces

$$
V(\gamma ; i)=\left\{v \in V \mid(\rho \circ \gamma)(t) v=t^{i} v, \forall t \in \mathbf{G}_{m}\right\}
$$

for $i \in \mathbf{Z}$. We write $X_{*}(A)$ for the set of cocharacters of $A$.

Consider now the reductive group $G$. If $\gamma \in X_{*}(G)$, then

$$
P_{G}(\gamma)=P(\gamma)=\left\{x \in G \mid \lim _{t \rightarrow 0} \gamma(t) x \gamma\left(t^{-1}\right) \text { exists }\right\}
$$

is a parabolic subgroup of $G$ whose Lie algebra is $\mathfrak{p}(\gamma)=\sum_{i>0} \mathfrak{g}(\gamma ; i)$. Moreover, each parabolic subgroup of $G$ has the form $P(\gamma)$ for some cocharacter $\gamma$; for all this cf. Spr 98, 3.2.15 and 8.4.5].

We note that $\gamma$ "exhibits" a Levi decomposition of $P=P(\gamma)$. Indeed, $P(\gamma)$ is the semidirect product $Z(\gamma) \cdot U(\gamma)$, where $U(\gamma)=\left\{x \in P \mid \lim _{t \rightarrow 0} \gamma(t) x \gamma\left(t^{-1}\right)=1\right\}$ is the unipotent radical of $P(\gamma)$, and the reductive subgroup $Z(\gamma)=C_{G}\left(\gamma\left(\mathbf{G}_{m}\right)\right)$ is a Levi factor in $P(\gamma)$; cf. Spr 98, 13.4.2].

Lemma 5. Let $P$ be a parabolic subgroup of $G$, let $L$ be a Levi factor of $P$, let $\gamma \in X_{*}(L)$ and assume that $P=P(\gamma)$. Then $L=Z(\gamma)$ and the image of $\gamma$ lies in the connected center of $L$. 
Proof. Let $R$ be the radical of $P$. Then the Levi factors of $P$ are precisely the centralizers of the maximal tori of $R$; cf. [Bo 91, Cor. 14.19]. Since the connected center of a Levi factor of $P$ evidently lies in $R$, we see that the connected center of each Levi factor is a maximal torus of $R$.

Now, the centralizer $L_{1}=Z(\gamma)$ is a Levi factor of $P$, so that $\gamma$ is a cocharacter of the connected center of $L_{1}$; in particular, the image of $\gamma$ lies in $R$. Moreover, since $L_{1}=Z(\gamma)$, the centralizer of the image of $\gamma$ in $R$ is a maximal torus $S$ of $R$. It follows that $S$ is the unique maximal torus of $R$ containing the image of $\gamma$.

Since the image of $\gamma$ lies in $L$ and in $R$, and since $L$ intersects $R$ in a maximal torus of $R$, it follows that $S=L \cap R$ so that $L=L_{1}$ as required.

2.4. Instability in invariant theory. Let $(\rho, V)$ be a linear representation [always assumed finite dimensional] of $G$, and fix a closed $G$-invariant subvariety $S \subset V$. We are going to describe a precise form - due to Kempf and Rousseau - of the Hilbert-Mumford criteria for the instability of a vector $v \in V$ under the action of $G$.

Let us first briefly describe our goal: given a Lie subalgebra $\mathfrak{h} \subset \mathfrak{g}=\operatorname{Lie}(G)$, fix a basis $\mathbf{X}=\left(X_{1}, \ldots, X_{d}\right)$ of $\mathfrak{h}$. If the $G$-orbit of $\mathbf{X}$ in $\bigoplus^{d} \mathfrak{g}$ is not closed - so that $\mathbf{X}$ is an unstable vector - the results of Kempf and Rousseau permit us to associate to $\mathbf{X}$ a unique parabolic subgroup $P_{\mathbf{X}}$; see Corollary 9 below. If $g \in G$ satisfies $\operatorname{Ad}(g) \mathfrak{h}=\mathfrak{h}$, one of our main objectives is to show that $g \in P_{\mathbf{X}}$. Using $g$, we get a new basis $\operatorname{Ad}(g) \mathbf{X}=\left(\operatorname{Ad}(g) X_{1}, \ldots, \operatorname{Ad}(g) X_{d}\right)$ of $\mathfrak{h}$, and generalities show that $P_{\operatorname{Ad}(g) \mathbf{X}}=g P_{\mathbf{X}} g^{-1}$. So we want to prove the equality $P_{\mathbf{X}}=P_{\operatorname{Ad}(g) \mathbf{X}}$; it will then follow that $g \in P_{\mathbf{X}}$, as desired.

Return now to our general setting: $V$ is any linear representation of $G$. For $v \in V$, put

$$
|V, v|=\left\{\lambda \in X_{*}(G) \mid \lim _{t \rightarrow 0} \rho(\lambda(t)) v \quad \text { exists }\right\} .
$$

Write $V=\bigoplus_{i \in \mathbf{Z}} V(\lambda ; i)$ as in (2.3.1), and write $v=\sum_{i} v_{i}$ with $v_{i} \in V(\lambda ; i)$. Then evidently

$$
\lambda \in|V, v| \Longleftrightarrow v_{i}=0 \quad \forall i<0 ;
$$

if $\lambda \in|V, v|$ then of course $\lim _{t \rightarrow 0} \rho(\lambda(t)) v=v_{0}$.

Now let $S \subset V$ be a $G$-invariant closed subvariety and suppose that $v \notin S$. Given $\lambda \in|V, v|$, write $v_{0}=\lim _{t \rightarrow 0} \rho(\lambda(t)) v$. If $v_{0} \in S$, write $\alpha_{S, v}(\lambda)$ for the order of vanishing of the regular function $\left(t \mapsto \rho(\lambda(t)) v-v_{0}\right): \mathbf{A}^{1} \rightarrow V$, otherwise write $\alpha_{S, v}(\lambda)=0$; see [Ke 78, $\left.\S 3\right]$ for more details. Then $\alpha_{S, v}(\lambda)$ is a non-negative integer, and $\alpha_{S, v}(\lambda)>0$ if and only if $v_{0} \in S$. Moreover, if $v=\sum_{i \in \mathbf{Z}} v_{i}$ with $v_{i} \in V(\lambda ; i)$ as before, then

$$
v_{0} \in S \Longrightarrow \alpha_{S, v}(\lambda)=\alpha_{\left\{v_{0}\right\}, v}(\lambda)=\min \left\{j>0 \mid v_{j} \neq 0\right\} .
$$

Suppose that $W \subset V$ is a subspace of dimension $d=\operatorname{dim} W$. Let $w_{1}, \ldots, w_{d}$ be a basis of $W$, and consider the point $x=\left(w_{1}, \ldots, w_{d}\right)$ of the linear space $X=\bigoplus^{d} V$; abusing notation somewhat, we write also $\rho$ for the diagonal action $\bigoplus^{d} \rho$ of $G$ on $X$. We observe for $\lambda \in X_{*}(G)$ that we have

$$
\lambda \in|X, x| \Longleftrightarrow W \subset \sum_{j \geq 0} V(\lambda ; j) .
$$

Fix $S \subset X=\bigoplus^{d} V$ a closed and $\rho(G)$-invariant subvariety, and assume that $x=$ $\left(w_{1}, \ldots, w_{d}\right) \notin S$. In this setting one may compute the function $\alpha_{S, x}$ for the diagonal $G$ action on $X$ using functions $\alpha_{\left\{v_{0}\right\}, v}$ for the $G$-representation $V$. More precisely, we have:

Lemma 6. Let $\lambda \in|X, x|$ and suppose $\alpha_{S, x}(\lambda)>0$. For $w \in W$, write $w_{0}=\lim _{t \rightarrow 0} \rho(\lambda(t)) w$. Then

$$
(*) \quad \alpha_{S, x}(\lambda)=\min _{w \in W} \alpha_{\left\{w_{0}\right\}, w}(\lambda) .
$$


Proof. For $1 \leq i \leq d$ write $x=\sum_{j} x^{j}$ with $x^{j} \in X(\lambda ; j)$.

By assumption, $\lambda \in|X, x|$; by (2.4.1) we see that $x^{j}=0$ if $j<0$. Moreover, using (2.4.2) we see that

$$
\alpha_{S, x}(\lambda)=\alpha_{\left\{x^{0}\right\}, x}(\lambda)=\min \left(j>0 \mid x^{j} \neq 0\right) .
$$

If we now write $R=\min _{v \in W} \alpha_{\left\{v_{0}\right\}, v}(\lambda)$ for the right hand side of $(*)$, then upon considering the components in $V$ of the vectors $x^{j} \in X=\bigoplus^{d} V$, one uses (2.4.4) to see that $\alpha_{S, x}(\lambda) \geq R$.

On the other hand, we may choose $v \in W$ such that $R=\alpha_{\left\{v_{0}\right\}, v}(\lambda)$. Writing $v=\sum_{j \geq 0} v^{j}$ with $v^{j} \in V(\lambda ; j)$, we see that

$$
R=\alpha_{\left\{v^{0}\right\}, v}(\lambda)=\min \left(j>0 \mid v^{j} \neq 0\right)
$$

by (2.4.1). Now write

$$
v=\sum_{i} \beta_{i} w_{i} \quad \text { for scalars } \beta_{i} \in K
$$

Now, $v^{R} \neq 0$ implies that $x^{R} \neq 0$; it follows from (2.4.4) that $R \geq \alpha_{S, x}(\lambda)$, and the Lemma is proved.

Fix a basis $\left\{w_{i}\right\}$ for $W$ and let $x=\left(w_{1}, \ldots, w_{d}\right) \in X$. Write

$$
S=\overline{\rho(G) x}-\rho(G) x
$$

then $S$ is closed in $X$ Notice that $S$ is a closed subset, since $\rho(G) x$ is open in $\overline{\rho(G) x}$, and $S$ is $G$-invariant. We suppose that $\rho(G) x$ is not closed, or equivalently that $S$ is non-empty.

Corollary 7. Let $h \in G$ satisfy $\rho(h) W=W$. If $x^{\prime}=\rho(h) x$, then we have $|X, x|=\left|X, x^{\prime}\right|$. Moreover,

for each $\lambda \in|X, x|$.

$$
\alpha_{S, x}(\lambda)=\alpha_{S, x^{\prime}}(\lambda)
$$

Proof. Since by (2.4.3) the sets $|X, x|$ and $\left|X, x^{\prime}\right|$ both consist of all cocharacters $\lambda$ for which $W \subset \sum_{j \geq 0} V(\lambda ; j)$, we have that $|X, x|=\left|X, x^{\prime}\right|$.

Now write $x_{0}=\lim _{t \rightarrow 0} \rho(\lambda(t)) x$ and $x_{0}^{\prime}=\lim _{t \rightarrow 0} \rho(\lambda(t)) x^{\prime}$. We first claim that $x_{0} \in S$ if and only if $x_{0}^{\prime} \in S$.

Well, assume that $x_{0} \notin S$. Since $x_{0}$ lies in the closure of $\rho(G) x$ but not in $S$, it actually lies in $\rho(G) x$; thus $(\dagger) x_{0}=\rho(g) x$ for some $g \in G$.

Since the components in $V$ of the vector $x \in X=\bigoplus^{d} V$ form a basis of $W$, one concludes from $(\dagger)$ that

$$
\lim _{t \rightarrow 0} \rho(\lambda(t)) y=\rho(g) y
$$

for each $y \in \bigoplus^{d} W \subset X$. This shows in particular that $x_{0}^{\prime}=\rho(g) x^{\prime}=\rho(g h) x$, so that $x_{0}^{\prime} \notin S$. Since the argument just given is symmetric in $x$ and $x^{\prime}$, it follows that $x_{0} \in S$ if and only if $x_{0}^{\prime} \in S$.

Recall that $\alpha_{S, x}(\lambda)>0$ if and only if $x_{0} \in S$ and that $\alpha_{S, x^{\prime}}(\lambda)>0$ if and only if $x_{0}^{\prime} \in S$. Thus to prove the final equality asserted by the corollary, we may suppose that $x_{0}, x_{0}^{\prime} \in S$. Now, according to $(*)$ of Lemma 6 we have

$$
\alpha_{S, x}(\lambda)=\min _{w \in W} \alpha_{\left\{w_{0}\right\}, w}(\lambda)=\alpha_{S, x^{\prime}}(\lambda)
$$

as required.

Fix a real-valued $G$-invariant length function $\lambda \mapsto\|\lambda\|$ on the set $X_{*}(G)$ of cocharacters of $G$. 
Theorem 8 (Kempf [Ke 78 Theorem 3.4], Rousseau). Let $z \in X-S$ and assume that $\overline{\rho(G) z} \cap S$ is non-empty. Then the function $\alpha_{S, z}(\lambda) /\|\lambda\|$ assumes a maximal value $B>0$ on the non-trivial elements of $|X, z|$. Let

$$
\Delta_{S, z}=\left\{\lambda \in|X, z| \mid \alpha_{S, z}(\lambda)=B \cdot\|\lambda\| \quad \text { and } \lambda \text { is indivisible }\right\} .
$$

Then

(1) $\Delta_{S, z}$ is non-empty,

(2) there is a parabolic subgroup $P_{S, z}$ of $G$ such that $P_{S, z}=P(\lambda)$ for each $\lambda \in \Delta_{S, z}$,

(3) $\Delta_{S, z}$ is a principal homogeneous space under $R_{u} P_{S, z}$, and

(4) any maximal torus of $P_{S, z}$ contains a unique cocharacter which lies in $\Delta_{S, z}$.

Let $H \subset G$ be a subgroup and suppose that $W$ is $\rho(H)$ invariant. Let $x=\left(w_{1}, \ldots, w_{d}\right) \in X$ for a basis $\left\{w_{i}\right\}$ of $W$.

Corollary 9. Assume that $\rho(G) x$ is not closed in $X$, and let

$$
S=\overline{\rho(G) x}-\rho(G) x .
$$

Then

(1) $P_{S, x}$ is a proper parabolic subgroup of $G$,

(2) $H \subset P_{S, x}$, and

(3) if $L \subset P_{S, x}$ is a Levi factor, there is a cocharacter $\lambda$ of the connected center $Z$ of $L$ which lies in $\Delta(S, x)$.

Proof. Since the image of any $\lambda \in|X, x|$ with $\alpha_{S, x}(\lambda)>0$ is not central in $G$, (1) is immediate.

Since the parabolic subgroup $P=P_{S, x}$ is self-normalizing, (2) will follow if we show that $h P h^{-1}=P$ for each $h \in H(k)$. But $h P_{S, x} h^{-1}=P_{S, \rho(h) x}$; see e.g. Ke 78, Cor. 3.5]. Since $\rho(h) W=W$, Corollary 7 shows that $|X, x|=|X, \rho(h) x|$ and that $\alpha_{S, x}(\lambda)=\alpha_{S, \rho(h) x}(\lambda)$ for all $\lambda \in|X, x|=|X, \rho(h) x|$; thus $\Delta_{S, x}=\Delta_{S, \rho(h) x}$ so that $P_{S, x}=P_{S, \rho(h) x}$ by Theorem 8 Thus indeed $H \subset P_{S, x}$.

Finally, for (3) let $S$ be a maximal torus of $L$ and hence of $P_{S, x}$. By (3) of Theorem $8 S$ has a cocharacter $\lambda$ which lies in $\Delta_{S, x}$. Since $P_{S, x}=P(\lambda)$, it follows from Lemma 5 that the image of $\lambda$ lies in the connected center of $L$, as required.

Finally, we record:

Lemma 10. Assume that $\rho(G) x$ is closed in $X$ and that $\lambda \in|X, x|$. Then the subset

$$
\lim _{t \rightarrow 0} \rho(\lambda(t)) W=\left\{\lim _{t \rightarrow 0} \rho(\lambda(t)) w \mid w \in W\right\}
$$

satisfies

$$
\lim _{t \rightarrow 0} \rho(\lambda(t)) W=\rho(g) W
$$

for some $g \in G$.

Proof. Since $\lambda \in|X, x|$, the limit $x_{\lambda}=\lim _{t \rightarrow 0} \rho(\lambda(t)) x$ exists. Since the orbit $\rho(G) x$ is closed, we have $\rho(g) x=x_{\lambda}$ for some $g \in G$. Since $w_{1}, \ldots, w_{d}$ is a basis of $W$, it follows that $\operatorname{Ad}(g) w=\lim _{t \rightarrow 0} \rho(\lambda(t)) w$ for each $w \in W$, whence the Lemma. 


\section{Proof OF THE MAIN THEOREM}

Recall that $G$ is a reductive group with Lie algebra $\mathfrak{g}$, and that $\mathfrak{h} \subset \mathfrak{g}$ is a Lie subalgebra. Fix a basis $X_{1}, \ldots, X_{d} \in \mathfrak{h}$, and let $\mathbf{X}=\left(X_{1}, \ldots, X_{d}\right) \in \bigoplus^{d} \mathfrak{g}=Y$. We write (Ad, $Y$ ) for the representation $\left(\bigoplus^{d} \mathrm{Ad}, \bigoplus^{d} \mathfrak{g}\right)$ of $G$.

Proof of part (1) of Theorem [1. Recall that we must show: the Lie algebra $\mathfrak{h}$ is $G$-cr if and only if the $G$-orbit of $\mathbf{X}$ is closed in $Y=\bigoplus^{d} \mathfrak{g}$.

We first suppose that $\operatorname{Ad}(G) \mathbf{X}$ is closed, and we show that $\mathfrak{h}$ is $G$-cr. Let $S$ be a maximal torus of the centralizer $C_{G}(\mathfrak{h})$. Then $\mathfrak{h} \subset \operatorname{Lie}(L)$ where $L=C_{G}(S)$; moreover, $L$ is a Levi factor of a parabolic subgroup of $G$. It follows from Lemma 4 that $\mathfrak{h}$ is $G$-cr if and only if $\mathfrak{h}$ is $L$-cr.

Moreover, it follows from Proposition 2 that $\operatorname{Ad}(L) \mathbf{X}$ is closed in $Y$. Thus we may replace $G$ by $L$ and so suppose that any torus in $G$ which centralizes $\mathfrak{h}$ is central in $G$. [Equivalently: $\mathfrak{h}$ is not contained in the Lie algebra of any Levi factor of a proper parabolic subgroup of $G$.] To show that $\mathfrak{h}$ is $G$-cr we will show that $\mathfrak{h}$ is not contained in $\operatorname{Lie}(P)$ for any proper parabolic subgroup $P$ of $G$.

Suppose that $\mathfrak{h} \subset \operatorname{Lie}(P)$ for a parabolic subgroup $P \subset G$; we will show that $P=G$. Write $P=P(\phi)$ for some cocharacter $\phi$ of $G$, and write $L=L(\phi)$ for the centralizer in $G$ of the image of $\phi$; then $L$ is a Levi factor of $P$.

Since the $G$-orbit of $\mathbf{X}$ is closed, Lemma 10 shows that

$$
\lim _{t \rightarrow 0} \operatorname{Ad}(\phi(t)) \mathfrak{h}=\operatorname{Ad}(g) \mathfrak{h}
$$

for some $g \in G$. Since $\lim _{t \rightarrow 0} \operatorname{Ad}(\phi(t)) H \in \operatorname{Lie}(L)$ for each $H \in \mathfrak{h}$, we conclude that $\mathfrak{h} \subset$ $\operatorname{Ad}\left(g^{-1}\right) \operatorname{Lie}(L)$. But then the image of the cocharacter $\operatorname{Int}\left(g^{-1}\right) \circ \phi$ is a torus centralizing $\mathfrak{h}$; hence the image of $\phi$ is central in $G$ so that $P=G$. This proves that $\mathfrak{h}$ is indeed $G$-cr.

To complete the proof of (i), it remains to show: if the orbit $\operatorname{Ad}(G) \mathbf{X}$ is not closed, then $\mathfrak{h}$ is not $G$-cr. As in Corollary 9 let $S=\overline{\operatorname{Ad}(G) \mathbf{X}}-\operatorname{Ad}(G) \mathbf{X}$; our assumption means that $S$ is non-empty so that $\alpha_{S, \mathbf{X}}(\lambda)>0$ for each $\lambda \in \Delta_{S, \mathbf{X}}$. Moreover, $P=P_{S, \mathbf{X}}$ is a proper parabolic subgroup of $G$.

We have $\mathfrak{h} \subset \operatorname{Lie}(P)$ by (2.4.3). To complete the proof, we suppose $\mathfrak{h}$ is $G$-cr, and find a contradiction.

Since $\mathfrak{h}$ is $G$-cr, there is a Levi factor $L$ of $P$ with $\mathfrak{h} \subset \operatorname{Lie}(L)$. By Corollary 9 there is a cocharacter $\lambda$ of the connected center of $L$ which lies in $\Delta_{S, \mathbf{X}}$. Since $\mathfrak{h} \subset \operatorname{Lie}(L)$, we have $\mathfrak{h} \subset \mathfrak{g}(\lambda ; 0)$; thus $\mathbf{X} \in X(\lambda ; 0)$. But then $\alpha_{S, \mathbf{X}}(\lambda)=0$, which is impossible since $\lambda \in \Delta_{S, \mathbf{X}}$.

Proof of part (2) of Theorem 1 Recall that if $H \subset G$ is a subgroup which is $G$-cr, we must prove that $\mathfrak{h}=\operatorname{Lie}(H)$ is $G$-cr.

Let $S \subset C_{G}(H)$ be a maximal torus. Then $H \subset L=C_{G}(S)$ and $\mathfrak{h} \subset \operatorname{Lie}(L)$. Applying Lemma 4 it is enough to show that $\mathfrak{h}$ is $L$-cr; thus we replace $G$ by $L$ and so suppose that $H$ is not contained in a Levi factor of any proper parabolic subgroup of $G$. Since $H$ is $G$-cr, we conclude that $H$ is contained in no proper parabolic subgroup of $G$.

To show that $\mathfrak{h}$ is $G$-cr, we use part (1) of Theorem 1 it is enough to show that $\operatorname{Ad}(G) \mathbf{X}$ is closed in $Y$. In fact, we are going to suppose that $\operatorname{Ad}(G) \mathbf{X}$ is not closed and obtain a contradiction. Let $S=\overline{\operatorname{Ad}(G) \mathbf{X}}-\operatorname{Ad}(G) \mathbf{X}$ and let $P=P_{S, \mathbf{X}}$. Since $S$ is assumed nonempty, Corollary 9 shows that $P$ is a proper parabolic subgroup. Moreover, since $\operatorname{Ad}(H)$ leaves $\mathfrak{h}$ invariant, that same corollary shows that $H \subset P$. This contradiction completes the proof. 


\section{REFERENCES}

[BMR 05] M. Bate, B.M.S. Martin, and G. Röhrle, A Geometric Approach to Complete Reducibility, Inv. Math. 161 (2005), $177-218$.

[Bo 91] Armand Borel, Linear Algebraic Groups, Graduate Texts in Math., Springer-Verlag, 1991.

[Ja 04] Jens Carsten Jantzen, Nilpotent orbits in representation theory, Lie Theory: Lie Algebras and Representations (J -P. Anker and B. Orsted, eds.), Progress in Mathematics, vol. 228, Birkhäuser, Boston, 2004, pp. 1-211.

[Ke 78] George R. Kempf, Instability in invariant theory, Ann. of Math. (2) 108 (1978), 299-316. MR 80c:20057

[Ma 03] Benjamin M. S. Martin, A normal subgroup of a strongly reductive subgroup is strongly reductive, J. Algebra 265 (2003), 669-674.

[MT 05] George J. McNinch and Donna M. Testerman, Completely reducible SL(2)-homomorphisms (2005), preprint.

[Ri 88] R. W. Richardson, Conjugacy classes of $n$-tuples in Lie algebras and algebraic groups, Duke Math. J. 57 (1988), 1-35.

[Ser 05] Jean-Pierre Serre, Complète Réductibilité, Astérisque 299 (2005), Exposés 924-937, pp. 195-217, Séminaire Bourbaki 2003/2004.

[Spr 98] Tonny A. Springer, Linear algebraic groups, 2nd ed., Progr. in Math., vol. 9, Birkhäuser, Boston, 1998.

Department of Mathematics, Tufts University, 503 Boston Avenue, Medford, MA 02155, USA

E-mail address: george.mcninch@tufts.edu 